„Beim Referentenentwurf handelt es sich lediglich um eine

Teilregelung, die rechtliche Unsicherheiten nur vergrößert

und die bestehenden Wertungswidersprüche nicht beseitigt."

\title{
Reformstau und kein Ende in Sicht
}

n Deutschland regelt seit dem 1.1.1991 das Embryonenschutzgesetz die Behandlungsmöglichkeiten der Reproduktionsmedizin. Richtlinien zu Behandlungsdetails werden üblicherweise von der Bundesärztekammer aufgestellt und durch die regionalen Ärztekammern in das Berufsrecht übernommen. Die Bundesärztekammer ist in regelmäBigen Abständen mit der dringenden Bitte an die politischen Entscheidungsträger herangetreten, sich der elementaren und dringlichen Aufgabe zu widmen, den offenen Fragen der Reproduktionsmedizin mit einer systematischen Rechtsentwicklung zu begegnen, so letztmalig anlässlich des 116. Deutschen Ärztetages in Hannover 2013. In den Koalitionsverhandlungen der gegenwärtigen Bundesregierung wurde vereinbart, das Recht auf Kenntnis der genetischen Abstammung zu regeln.

Jetzt liegt endlich der langersehnte Entwurf eines Gesetzes zur Regelung des Rechts auf Kenntnis der Abstammung bei donogener (heterologer) Verwendung von Samen vor. Dies ist an sich eine erfreuliche Entwicklung: Durch Einrichtung eines Zentralregisters soll in Zukunft sichergestellt werden, dass Spenderkinder die Daten ihres genetischen Erzeugers ausgehändigt bekommen. Weiterhin soll der Spender von sämtlichen Verpflichtungen (Unterhaltsrecht/Erbrecht) freigestellt werden und auf keinen Fall als Vater eines donogen gezeugten Kindes festgestellt werden können.

Insgesamt ein positiver Ansatz, der jedoch bei näherer Überprüfung nicht standhält. Werden doch durch den Entwurf nur die Kinder erfasst, die durch eine medizinische donogene Behandlung entstanden sind. Die heute schon viel größere Gruppe der nicht mit medizinischer Hilfe (sogenannte BecherMethode) und der durch Bezug von Sperma aus dem Ausland und Home-Insemination erzeugten Schwangerschaften wird durch diese Regelung nicht erfasst. Auch werden die vermutlich mehr als 1.000 durchgeführten Eizellspenden in die Regelungen nicht miteinbezogen, sodass weiterhin die Mehrzahl der Kinder keine Möglichkeit hat, ihre geneti- sche Abstammung in Erfahrung zu bringen. Die Bundesärztekammer kritisiert daher den Entwurf des Gesundheitsministeriums auch vehement als Stückwerk.

Die offenen, insbesondere die gesellschaftspolitischen und familienrechtlichen Fragen bezüglich der donogenen Samenspende werden mit dem vorliegenden, im Wesentlichen auf die Einrichtung eines Samenspenderregisters, die entsprechenden Informations- und Meldepflichten sowie datenschutzrechtliche Regelungen fokussierten Referentenentwurf weiterhin nicht ausreichend beantwortet. Es handelt sich hier lediglich um eine Teilregelung, welche die rechtlichen Unsicherheiten nur vergrößert und die bestehenden Wertungswidersprüche nicht beseitigt.

Darüber hinaus hat die Arbeitsgruppe „Abstammung“, die beim Justizministerium angesiedelt ist, erste Ergebnisse erst für den Sommer 2017 versprochen, sodass wir von einem modernen Fortpflanzungsmedizingesetz nach wie vor weit entfernt sind.

Österreich hat hier viel schneller reagiert. In Reaktion auf eine Erkenntnis des Verfassungsgerichtshofes aus dem Jahr 2013, durch die es lesbischen Paaren freigestellt worden war, künstliche Insemination und In-vitro-Fertilisation (IVF) in Anspruch zu nehmen, kam es in Österreich zu einem Paradigmenwechsel im Recht der Fortpflanzungsmedizin. Dort wurde im Januar 2015 das Fortpflanzungsmedizinrechtsänderungsgesetz erlassen, das schon am 24.2.2015 in Kraft trat. In Deutschland sind wir immer noch weit davon entfernt.

Regelungen, wie mit den Folgen der Reproduktionsmedizin praktisch umzugehen ist, müssen nach wie vor von den Gerichten geklärt werden.

Das ist ein unhaltbarer Zustand.

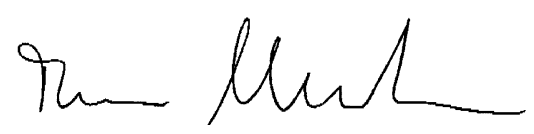

